\title{
Evaluation of popliteal cysts and painful calves with ultrasonography: comparison with arthrography
}

\author{
B. M. GOMPELS ANDL. G. DARLINGTON \\ From the Radiology Department and Rheumatology Unit, Epsom District Hospital, Epsom, Surrey
}

SUMMARY Grey-scale ultrasonography will detect reliably the presence of clinically significant popliteal cysts, fluid collections which do not fill by arthrography, and will frequently demonstrate ruptured cysts and the soft tissue changes resulting from a recent leak. Forty-eight knees, in 25 patients with popliteal and/or calf pain were examined by ultrasonography followed by arthrography. Popliteal cysts were demonstrated in $40 \%(19 / 48)$ by ultrasound and in $46 \%(22 / 48)$ by arthrogram. For comparison between arthrography and ultrasonography $\chi^{2}=8.58$ and contingency coefficient, $\Phi=0.42(\mathrm{p}<0.01)$. Acute cyst rupture was shown in 2 patients $(8 \%)$ by both arthrography and ultrasound. In a further study ultrasonography demonstrated popliteal cysts with a prevalence of $31 \%(22 / 72)$ in 36 patients with definite or classical rheumatoid arthritis compared with $4 \%$ (3/72) in controls closely matched for age and sex. This difference in prevalence between the rheumatoid patients and controls was highly significant $\chi^{2}=17 \cdot 48, p<0 \cdot 001$. Ultrasonography, therefore, will demonstrate noninvasively the presence of popliteal cysts, may assist in the diagnosis of rupture, and furthermore will assist in quantitative, sequential assessment of patients with painful knees and calves.

A popliteal cyst is an abnormal distension of the gastrocnemio-semimembranosus bursa. Connection with the knee joint has been reported in about $50 \%$ of cadaveric knees ${ }^{1}$ and in $65 \%$ of excised cysts. ${ }^{2}$ Popliteal cysts may be associated with rheumatoid arthritis, ${ }^{3}$ gout, ${ }^{4}$ Reiter's syndrome, Sjögren's syndrome, ${ }^{5}$ ankylosing spondylitis, gonorrhoeal arthritis, ${ }^{6}$ degenerative arthritis, tears of the medial meniscus, and knees containing calcific deposits. Popliteal cysts may be symptomless ${ }^{8}$ or clinically undetectable. ${ }^{9}$ They may cause pain and impair knee function, may dissect into the calf, or may rupture. Hughes and Pridie ${ }^{10}$ suggested that cysts may also form after rupture by loculation of extravasated synovial fluid. Normally cysts disappear when the knee is extended, their synovial fluid being forced back into the joint space and suprapatellar pouch. ${ }^{11} \mathrm{~A}$ valvular mechanism has been suggested as a possible reason for persistence of cysts on extension ${ }^{12}$ with a potential for enlargement and/or rupture. Rauschning, ${ }^{13}$ however, in a study of necropsy specimens of knee joints, did not find a unidirectional valve mechanism but noted communication between knee

Accepted for publication 31 July 1981.

Correspondence to Dr B. M. Gompels, Epsom District Hospital, Dorking Road, Epsom, Surrey KT18 7EG. joint and bursa in $54 \%$ of knees, opening and closing according to tendon action.

The clinical presentation of cyst rupture, of a large unruptured cyst, and of deep venous thrombosis may all be similar. ${ }^{8}$ Precise diagnosis is important, since inappropriate anticoagulation may cause bleeding into the calf and prolonged, difficult recovery from muscle contracture. ${ }^{14}$

Ultrasonic scanning has been used to demonstrate popliteal cysts ${ }^{15}$ and to investigate painful calves. ${ }^{16}$ Meire et al. ${ }^{17}$ and Ambanelli et al. ${ }^{18}$ emphasised its ability to demonstrate cysts which do not fill on arthrography.

The aims of this paper are 3-fold: firstly, to reaffirm, by comparison with arthrography, the use of grey-scale ultrasonography as a reliable, painless, noninvasive means of detecting popliteal cysts of clinically significant size and of detecting cyst rupture, as we have described elsewhere ${ }^{19}$; secondly, to compare the prevalence of popliteal cysts detected by ultrasound in patients with definite and classical rheumatoid arthritis with the prevalence of such cysts in age- and sex-matched controls without symptomatic arthropathy; thirdly, to demonstrate the ability of grey-scale ultrasonography to assist in sequential studies of patients with popliteal cysts and/or painful calf lesions. 


\section{Comparison with arthrography}

\section{Material and method}

Twenty-five patients with popliteal cysts and/or calf pain were examined clinically to detect palpable popliteal cysts and were then investigated by ultrasonography followed by arthrography. Eight patients had classical or definite rheumatoid arthritis by the American Rheumatism Association criteria ${ }^{20} ; 4$ had seronegative, inflammatory arthropathies; one had gout; and the remainder had degenerative, traumatic, or pyrophosphate arthropathies. Serial, longitudinal ultrasound scans of the popliteal space and calf were obtained with the patient prone and the leg extended. By a standard arthrographic technique 20 $\mathrm{ml}$ of Conray 280 was injected into the knee and 20 full flexion and extension knee movements performed. Anteroposterior and lateral knee radiographs were taken in full extension with the patient supine. All arthrograms and ultrasound films were interpreted at the time of examination and then subsequently on 2 further occasions, one week apart, blindly and in a different order, to minimise observer error.

\section{Results}

In $46 \%$ of knees $(22 / 48)$ popliteal cysts were demonstrated by arthrography and in $40 \%(19 / 48)$ by ultra- sonic scanning. Cysts were revealed by ultrasonography alone in $10 \%(5 / 48)$ and arthrography alone in $17 \%(8 / 48)$ knees. Ultrasonography gave a small number of false negative results, $3 / 49(6 \%)$, usually those in which the diameter of the cyst was less than $0.5 \mathrm{~cm}$ and which were therefore of doubtful clinical significance. Clinical examination gave a larger number of false negative results, $8 / 49(16 \%)$. Neither clinical examination nor ultrasonography gave false positive results and so were never positively clinically misleading.

A $\chi^{2}$ test was applied to determine the significance of association between arthrography (as the reference method) and the alternative methods, that is, ultrasonography or clinical assessment. In addition, a contingency coefficient, $\Phi$, was determined. For comparison between arthrography and ultrasonography $\chi^{2}=8.58$ and $\Phi=0.42(\mathrm{p}<0.01)$. For comparison between arthrography and clinical assessment $\chi^{2}=1.15$ and $\Phi=0.15$ ( $\mathrm{p}$ not significant).

When arthrography was also used as the reference technique for detection of cyst rupture, ultrasonic scanning gave 4 false negative and one false positive results, while clinical examination gave 5 false positive results.

Both arthrography and ultrasonography showed synovial thickening and/or fibrin deposition of 'melon seed' bodies, and posterior bulging of the normal joint capsule was seen in 16 extended knees.

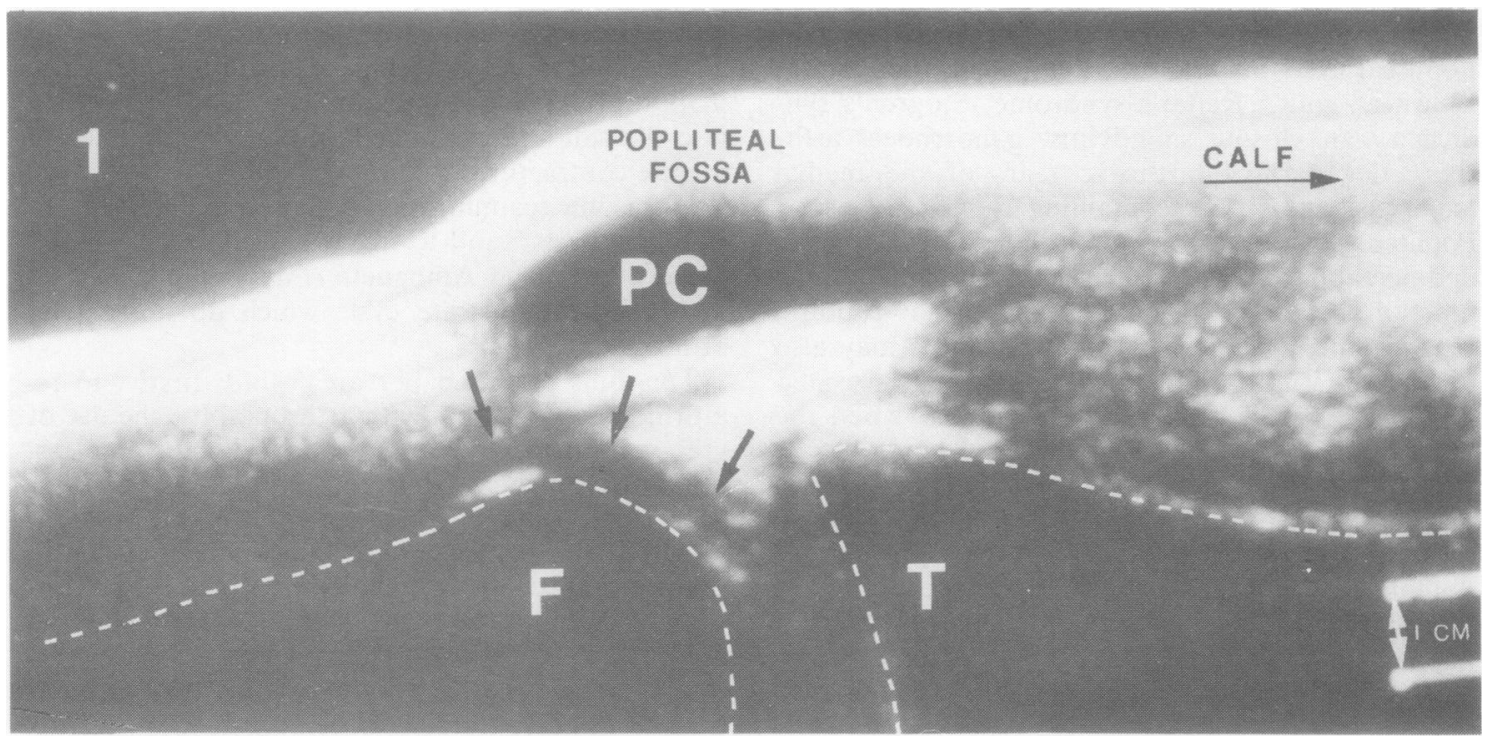

Fig. 1 Prone lateral sectional view of popliteal fossa with unruptured popliteal cyst (PC). The 3 mm thick curvilinear black band (arrowed) represents the posterior extension of a small knee effusion paralleling the posterior aspect of the femoral condyle (F). $\mathrm{T}=$ tibia. 
In 10 knees there was a meniscus of fluid (Fig. 1) paralleling the posterior aspect of the tibial and femoral condyles due to the presence of a joint effusion.

A diagnosis of cyst rupture was confirmed in 2 patients by both ultrasonography and arthrography. In one of these (Fig. 2) the leak of fluid from the cyst was noted on ultrasonic scanning to have extended more distally and superficially than had been suspected arthrographically. In 4 knees the ultrasonic diagnosis of rupture appeared probable from the attenuated appearance of the lower margin of the cysts, and in 3 of these cases soft tissue changes distal and adjacent to the cyst were shown (Fig. 3).

In 6 patients no cause for pain was revealed by either arthrography or ultrasonography, but one

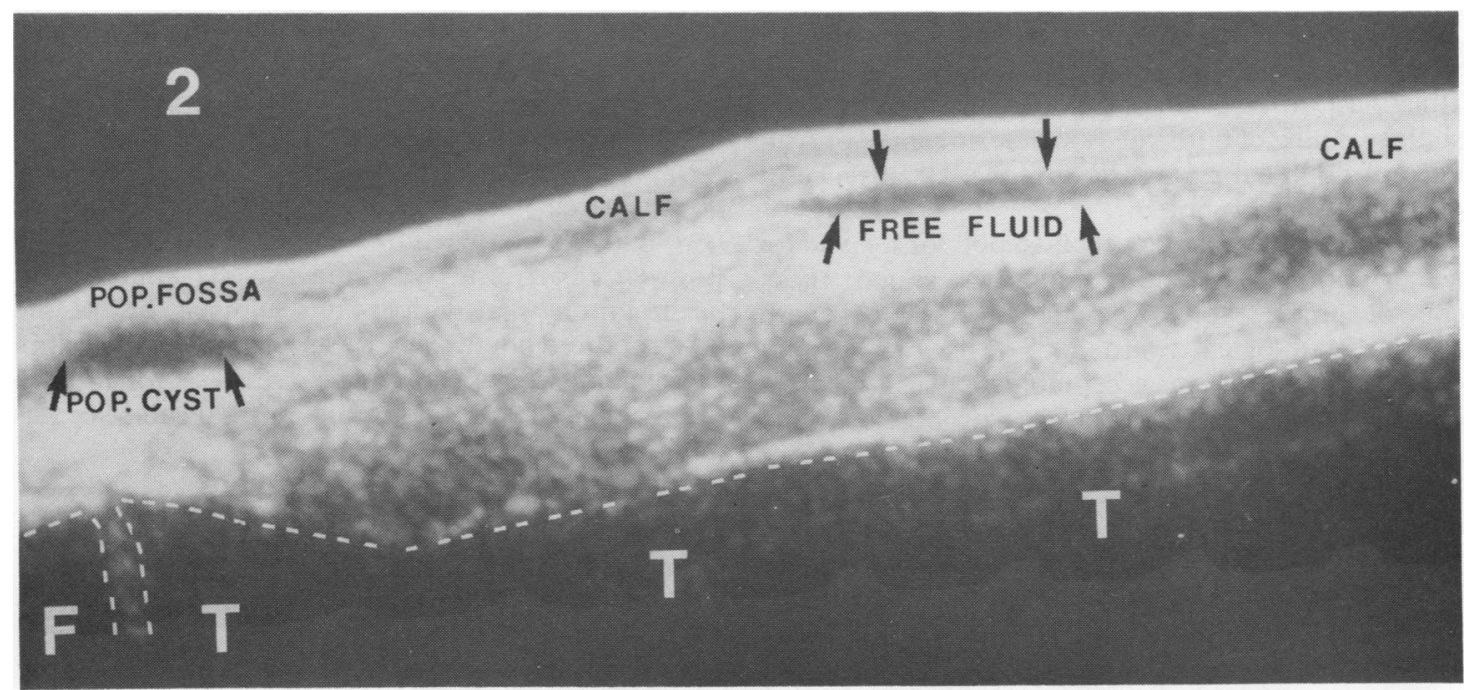

Fig. 2 Prone lateral projection of knee and calf. Rupture of the popliteal cyst produced a superficial fluid collection, not outlined by arthrography but seen with ultrasound to extend distally to the ankle. This fluid had been reabsorbed completely 3 months after rupture. $\mathrm{F}=$ femur. $\mathrm{T}=$ tibia.

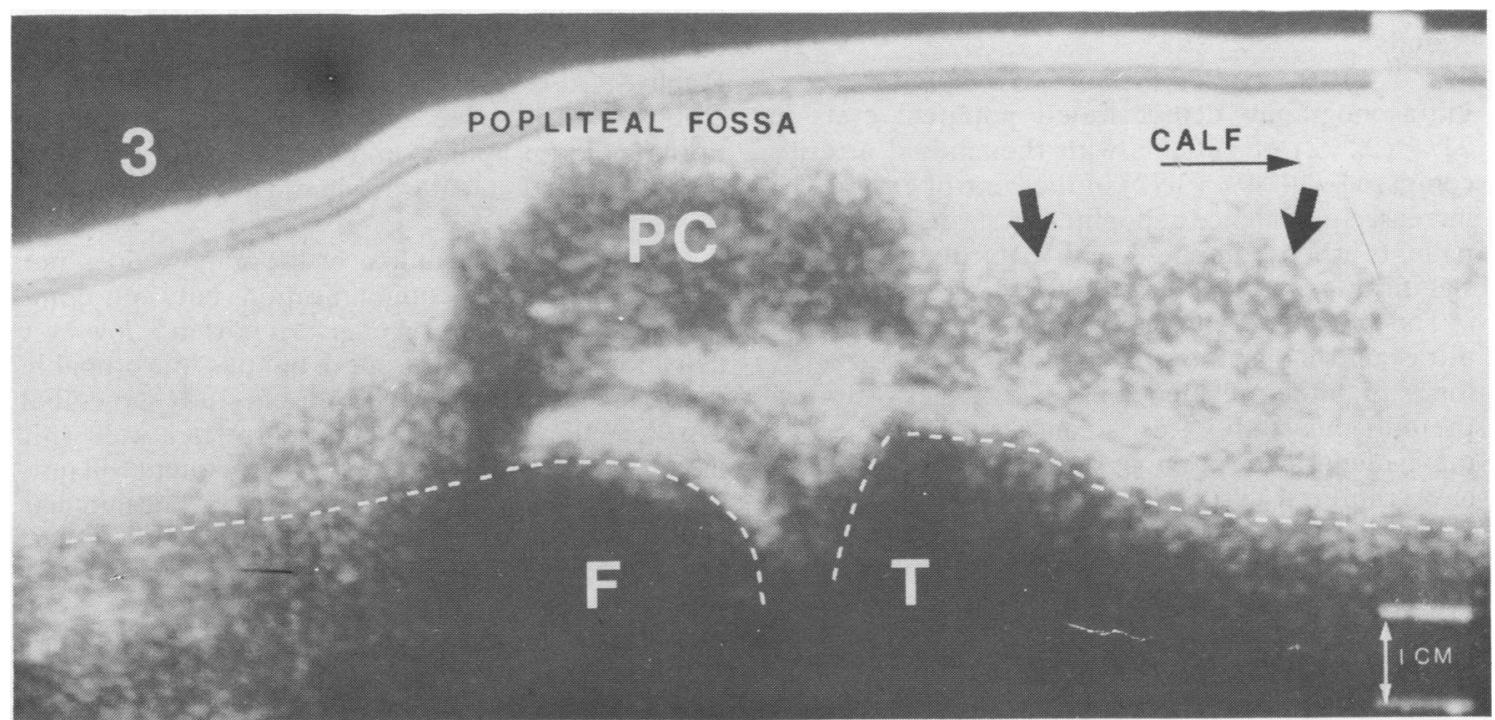

Fig. 3 Recent rupture of a popliteal cyst (PC). Prone lateral projection illustrating soft tissue changes (arrowed) distal to the cyst. The cyst has a more mottled appearance due to an echo response to debris within it. $\mathrm{F}=$ femur. $\mathrm{T}=$ tibia. 
patient with negative findings was subsequently found to have a torn calf muscle and 2 had deep venous thromboses.

\section{Prevalence of popliteal cysts in patients with rheumatoid arthritis compared with controls}

\section{Material and method}

Thirty-six patients ( 30 women and 6 men) with classical or definite rheumatoid arthritis by the American Rheumatism Association criteria ${ }^{20}$ underwent ultrasonic scanning of both knees to determine the prevalence of popliteal cysts. The presence or absence of effusions was also noted.

Thirty-six controls, closely matched for sex and for age within 5 years, were similarly scanned; these control subjects were also patients (in this case with nonrheumatological disorders). The use of a hospital control population was acceptable to assess the presence of an anatomical structure in a proportion of the general population, its presence or absence being unaffected by hospital admission. Since it was possible that admission and rest might make the cyst less full of fluid and less easily demonstrated, 20 full flexion-extension knee movements were applied to all subjects to avoid variations in prescan physical activity.

Thirty-one $(86 \%)$ of the rheumatoid patients were seropositive compared with only $1(3 \%)$ of the controls who had a positive RA latex test.

The prevalence of cysts in the rheumatoid and control populations was determined and compared.

\section{Results}

Ultrasonography demonstrated popliteal cysts in $31 \%(22 / 72)$ of patients with rheumatoid arthritis compared with $4 \%(3 / 72)$ of the control group. The increased prevalence of popliteal cysts in rheumatoid patients when compared with controls was highly significant, $\chi^{2}=17 \cdot 48, \mathrm{p}<0 \cdot 001$.

The prevalence of popliteal cysts detected with ultrasound in this study, that is, $22 / 72$ knees $(31 \%)$, is lower than the prevalence of $19 / 48$ knees $(40 \%)$ in the previous study. This is consistent with the fact that patients in the previous study were more likely to have ruptured cysts, since they presented acutely with pain.

\section{Sequential studies}

Ultrasonic scanning is invaluable in the quantitative, sequential assessment of patients with popliteal cysts or painful calves for whom a clinician would hesitate to request repeated arthrograms.
For example, patient A, a 68-year-old man with active seropositive rheumatoid arthritis presented with pain and restriction in knee movement. Ultrasonography revealed a $2 \mathrm{~cm}$ anteroposterior diameter popliteal cyst which caused at least some of his knee symptoms. The knees were aspirated and injected with steroid and the patient was given penicillamine, to which he responded well. A few months later a further ultrasonic scan showed a much smaller cyst containing little fluid-findings which coincided with his clinical improvement.

Patient B, an 80-year-old woman, presented with an acutely painful swollen calf. An ultrasonic scan (Fig. 2) revealed a popliteal cyst with an attenuated lower margin, suggestive of rupture, and within the swollen calf a collection of fluid. Thus ultrasound showed clearly, safely, noninvasively, and without arthrography that the patient's symptoms were due to a ruptured popliteal cyst. Serial scans of the calf in succeeding weeks showed progressive reduction in the volume of calf fluid until a normal calf scan was obtained.

\section{Discussion}

During arthrography contrast material sometimes may not move posteriorly to outline a cyst, particularly when patient co-operation is compromised by pain or when communication between cyst and knee joint is obstructed by loose bodies. ${ }^{21}$ When no communication between the knee joint and bursae exists, the entire posterior joint compartment may bulge to give a false impression of a cyst. Furthermore, a normal communicating bursa may be mistaken for a pathological cyst ${ }^{22}$ or may simulate a cyst on arthrography. Arthrography demonstrates synovial proliferation, the presence and approximate number of fibrinous loose bodies, and extensions of the joint space-important features when synovectomy is considered.

Ultrasonography, unlike arthrography, does not rely on persistent communication between joint space and cyst. Simpson et al. ${ }^{16}$ reported a low cyst detection rate with ultrasound, but this was probably due to technical factors. The present study shows that cyst detection with ultrasound correlates well with their detection by arthrography. Ultrasound will not, however, reliably detect cysts with an intraluminal anteroposterior diameter of much less than $0.5 \mathrm{~cm}$, and if the cyst is filled with debris it may not be seen very easily.

An intact calf cyst has a discrete margin, but joint rupture may be shown on arthrography by the feathery appearance of contrast spreading between muscles. This may be less obvious if arthrography is delayed or if the site of the rupture has closed. In time 
an inflammatory reaction develops which may protect the leg from further leakage. As in one of our cases, Moore et al. ${ }^{23}$ described a superficial cyst, not demonstrated by arthrography, from which was aspirated heavily blood-stained fluid. Arthrography therefore may fail to demonstrate a leak when ultrasonography may show the fluid collection appearing quite separately from the main popliteal cyst, with evidence of local, intense, soft tissue reaction. Among our cases all such isolated fluid collections were superficial, and, where rupture had occurred, the distal end of the parent cyst appeared to be attenuated and ill defined. Contrary to the findings of Meire et al. ${ }^{17}$ and Carpenter et al., ${ }^{21}$ we suggest that ultrasonography is now capable of indicating recent cyst rupture.

In prevalence studies seeking popliteal cysts in a population which is fit and symptom-free, ultrasound is ideal, since it enables an accurate measurement of cyst size to be performed safely at all stages of treatment. Ultrasound usually can detail noninvasively a ruptured popliteal cyst without resorting to arthrography but is sometimes nonspecific. This is especially relevant when significant oedema of the calf is present, and a further comparative study has been begun to assess whether ultrasound is capable of differentiating between deep venous thrombosis and ruptured popliteal cysts in patients presenting with painful calves.

\section{References}

${ }^{1}$ Wilson P D, Eyre-Brook A L, Francis J D. A clinical and anatomical study of the semimembranous bursa in relation to popliteal cyst. J Bone Joint Surg 1938; 20: 963-84.

2 Burleson R J, Bickel W H, Dahlin D C. Popliteal cyst. A clinicopathological study.J Bone Joint Surg 1956; 38A: 1267-74.

3 Meyerding H W, Van Denmark R E. Posterior hernia of the knee. JAMA 1943; 122: 858-61.

- Levitin P M, Keats T E. Dissecting synovial cysts of the popliteal space in gout. $A J R$ 1975; 124: 32-3.

s Gerber N J, Dixon A St J. Synovial cysts and juxta-articular bone cysts (Geodes). Semin Arthritis Rheum 1974; 3: 323-48.
- Baumann D, Kremer H. Arthrography and sonography in the diagnosis of Baker's cysts. ROEFO 1977; 127: 463-6.

7 Huskisson E C, Dieppe P A, Tucker A K, Canell L B. Another look at osteoarthritis. Ann Rheum Dis 1979; 38: 423-8.

- Hall A P, Scott J T. Synovial cysts and rupture of the knee joint in rheumatoid arthritis. An arthrographic study. Ann Rheum Dis 1966; 25: 32-41.

- Palmer D G. Synovial cysts in rheumatoid disease. Ann Intern Med 1969; 70: 61-8.

10 Hughes G R, Pridie R B. Acute synovial rupture of the knee-a differential diagnosis from deep vein thrombosis. Proc R Soc Med 1970; 63: 587-90.

1 Russell E, Hamm R, Le Page J T, Schoenbaum S W, Satin R. Some normal variations of knee arthrograms and their anatomical significance. J Bone Joint Surg 1978; 60A: 66-74.

12 Jayson M I V, Dixon A St J. Valvular mechanisms in juxtaarticular cysts. Ann Rheum Dis 1970; 29: 415-20.

13 Rauschning W. Anatomy and function of the communication between knee joint and popliteal bursae. Ann Rheum Dis 1980; 39: 354-8.

14 Tait G B W, Bach F, Dixon A St J. Acute synovial rupture. Ann Rheum Dis 1965; 25: 273-7.

15 McDonald D G, Leopold G R. Ultrasound B-scanning in the differentiation of Baker's cyst and thrombophlebitis. Br J Radiol 1972; 45: 729-32.

${ }^{16}$ Simpson F G, Bark M, Robinson P J, Losowsky M S. Prospective study of thrombophlebitis and 'pseudothrombophlebitis'. Lancet 1980; i: 331-3.

17 Meire H B, Lindsay D J, Swinson D R, Hamilton E B D. Comparison of ultrasound and positive contrast arthrography in the diagnosis of popliteal and calf swellings. Ann Rheum Dis 1974; 33: 221-4.

18 Ambanelli U, Manganelli P, Nervetti A, Ugolotti U. Demonstration of articular effusions and popliteal cysts with ultrasound. J Rheumatol 1976; 3: 134-9.

10 Gompels B M, Darlington L G. Grey-scale ultrasonography and arthrography in evaluation of popliteal cysts. Clin Radiol 1979; 30: $539-45$.

20 Ropes M W, Bennett G A, Cobb S, Jacor R, Jessar R A. Diagnostic criteria for rheumatoid arthritis. Ann Rheum Dis 1959; 18: 49-53.

${ }^{21}$ Carpenter J R, Hattery R R, Hunder G G, Bryan R S, McLeod R A. Ultrasound evaluation of the popliteal space. Comparison with arthrography and physical examination. Mayo Clin Proc 1976; 51: 498-503.

22 Doppman J L. Baker's cyst and the normal gastrocnemiussemimembranous bursa. AJR 1965; 94: 646-52.

${ }^{23}$ Moore C P, Sarti D A, Louie J S. Ultrasonographic demonstration of popliteal cysts in rheumatoid arthritis. A non-invasive technique. Arthritis Rheum 1975; 18: 577-80. 